\title{
Structural Organization of the Cytoskeleton in SV40 Human Corneal Epithelial Cells Cultured on Nano- and Microscale Grooves
}

\author{
Nancy W. Karuri ${ }^{1}$, Paul F. Nealey ${ }^{1}$, Christopher J. Murphy ${ }^{2}$, and Ralph M. Albrecht ${ }^{3}$ \\ ${ }^{1}$ Department of Chemical and Biological Engineering, University of Wisconsin, Madison, \\ Wisconsin \\ ${ }^{2}$ Department of Surgical Sciences, School of Veterinary Medicine, University of Wisconsin, \\ Madison, Wisconsin \\ ${ }^{3}$ Department of Animal Sciences, University of Wisconsin, Madison, Wisconsin
}

\section{Summary}

The basement membrane of human corneal epithelial cells (HCECs) has a three-dimensional nanoscale architecture, which includes pores, bumps and fibers that may influence cell-substrate adhesion and spreading in the overlying cells. We previously demonstrated that nano- and microscale groove and ridge patterns influence the morphological response and the adhesive response of HCECs to a nominal wall shear stress. Cell-substrate adhesion is mediated by adhesion receptors that bind to extracellular matrix components and anchor the cytoskeleton (CSK) of cells to extracellular elements. Here we investigate the CSK organization in SV40transformed HCECs grown on nano- and microscale groove and ridge patterns. X-ray lithography was used to fabricate uniform groove and ridge patterns with features ranging in size from $200 \mathrm{~nm}$ to $2 \mu \mathrm{m}$ grooves. Scanning electron microscopy and transmission electron microscopy were used to investigate CSK structure and the distribution of $-\beta 1$ integrin adhesion receptors. CSK elements aligned with the patterns; however, the spatial organization of these elements was influenced by feature size. Larger CSK bundles lay on top of the ridges and ran parallel to the patterns, whereas smaller CSK bundles, whose width was proportional to the groove size, spanned the grooves. $-\beta 1$ integrins co-localized with the CSK and had a higher density at the poles of aligned spindle-shaped cells. Differences in organization seen on the different topographical feature sizes may be indicative of differences in extracellular matrix organization. This may explain, in part, previous observations regarding the dependence of cell adhesive responses on the size of topographic features in the substrate.

\section{Keywords}

adhesion; cornea; cytoskeleton; epithelium; filopodia; holes; morphology; nanoscale; pattern; proliferation; topography

\section{Introduction}

Topography is one of many physical cues that cells encounter in their natural environment and it may play an integral role in ensuring the normal functioning of cells. Human corneal

Address for reprints: Ralph M. Albrecht, Department of Animal Sciences, 1675 Observatory Dr, University of Wisconsin, Madison, WI 53706, Albrecht@ansci.wisc.edu. 
epithelial cells (HCECs) lie on a basement membrane, which possesses a complex threedimensional nanoscale topography (Abrams et al. 2000, 2002). Basement membranes have been implicated in wound healing, embryonic development and in the development of the nervous system (Hieda and Nakanishi 1997; Leivo and Wartiovaara, 1989; Linsenmayer $e t$ al. 1998). A better understanding of how topography modulates fundamental cell functions would contribute significantly to the knowledge of important cell functions such as tissue repair, proliferation, differentiation and growth.

A variety of cells cultured on groove and ridge patterns have been observed to exhibit contact guidance (reviewed by Abrams et al. 2002). Contact guidance is characterized by an alignment and elongation and in some cases, migration of cells in the direction of the underlying patterns. Such changes in cell morphology have been associated with changes in cell-cycle progression and apoptosis (Chen et al. 1997; Huang et al. 2006). Studies have also shown that topography influences the orientation and size of focal adhesions (Teixeira et al. 2003). Focal adhesions are supramolecular complexes composed of integrins, adhesion and signaling molecules and are important for adhesion-mediated signaling (Pankov et al. 2003; Yamada et al. 2003). They serve as a basis for transduction of mechanical stimuli between integrins and the cytoskeleton (CSK).

In addition to binding extracellular matrix components, integrins help assemble and organize the extracellular matrix. For instance, the assembly of fibronectin, a major component of the extracellular matrix, into a fibrillar matrix is dependent on the binding and translocation of $-\beta_{1}$ integrins (Danen et al. 2002; Pankov et al. 2000). These interactions between integrins and the extracellular matrix are thought to expose cryptic binding sites in extracellular matrix allowing for the growth and development of this structure. Studies using fibroblasts cultured on microscale grooves have shown that cells exhibiting contact guidance on these surfaces produce more fibronectin than cells cultured on planar surfaces (Chou et al. 1995). A number of studies have proposed that the edges of synthetic substrates may be areas clustering of adhesion molecules. Condensations of actin and vinculin receptors have been observed at the edges of microscale grooves (Oakley and Brunette 1993; Wojciak-Stothard et al. 1995). These studies demonstrate that topography may influence a number of biochemical processes. However, currently additional evidence is required in order to more specifically determine the specific mechanisms by which topography influences adhesion receptors and the CSK.

Here we investigate how the structure of the CSK in SV40-HCECs, cultured on nano- and microscale grooves, is influenced by substrate topography. X-ray lithography was used to fabricate substrates with uniform grooves and ridges having a groove to ridge ratio of 1:1 and a pitch or periodicity of 400-4,000 $\mathrm{nm}$. Immunogold labeling was utilized in conjunction with scanning electron microscopy (SEM) and transmission electron microscopy (TEM) to examine the ultra-structure of the CSK and the distribution of $-\beta_{1}$ integrins at the basal surface of the cells. We chose to examine the distribution of $-\beta_{1}$ integrins because they interface between the CSK and the substrate via interactions with the extracellular matrix and they are in high abundance in adherent cells. The topographies used in this study have previously been shown to elicit a morphological and adhesive response in HCECs (Karuri et al. 2004; Teixeira et al. 2003) and, therefore, the correlative structural data obtained here provide a basis to more fully understand the morphologic and functional status associated with different substrate structures. 


\section{Materials and Methods}

\section{Substrate Preparation}

$\mathrm{X}$-ray lithography was used to fabricate single silicon chips containing six 4-mm² regions patterned with evenly spaced grooves varying in pitch or periodicity from nano- to microscale. First, the silicon wafers were cleaned and primed with hexamethyldisilazane (Yield Engineering Systems, San Jose, CA) to promote resist adhesion and then spin coated with UV6 photoresist (Shipley, Malborough, MA) in a resist spinner (Solitec Wafer Processing, Inc., San Jose, CA). The thickness of the resist structures was approximately $0.40 \mu \mathrm{m}$. The photoresist was then patterned on an X-ray Suss XRS-200/2M Stepper (Center for Nanotechnology, University of Wisconsin-Madison, WI). After patterning, a post exposure bake was performed on the resist by heating the wafer at $130{ }^{\circ} \mathrm{C}$ for $60 \mathrm{~s}$ on a hot plate. The exposed and baked films were then immersed in developing solution (MF320, Shipley) for $60 \mathrm{~s}$ to produce patterns on the resist-coated wafer consisting of exposed regions. Finally, a helicon-etching tool (Center for Plasma Aided Manufacturing, University of Wisconsin-Madison) was used to create the groove and ridge features on the silicon surfaces. The remaining resist was removed after etching by immersing the wafers in piranha solution $\left(70 \%\right.$ vol $\mathrm{H}_{2} \mathrm{SO}_{4}, 30 \%$ vol $\left.\mathrm{H}_{2} \mathrm{O}_{2}\right)$ at $110^{\circ} \mathrm{C}$ for $30 \mathrm{~min}$ and rinsing repeatedly with deionized water.

SEM was used to characterize the structure of the substrates. We used a LEO 1530 electron microscope (Leo Electron Microscopy Inc., Thornwood, NY) operating at $5 \mathrm{kV}$ and integrated with image analysis software. We measured the pitch or periodicity of the structures, the groove and ridge width as well as the depth of the structures (Fig. 1). The pitch dimensions defined the topographical areas and within each chip there were seven topographical areas: the $400,800,1,200,1,600,2,000,4,000 \mathrm{~nm}$ pitch dimensions and the planar surface. For ease of reference, the patterns will be referred to hereafter in terms of the pitch size. The planar surface was used as the control for comparison of cell behavior on patterned surfaces.

\section{Cell Culture}

Simian virus-transformed HCECs (SV40-HCECs, generously provided by Dr. Araki-Sasaki, Kiniki Central Hospital, Hyogo, Japan) were cultured to 90-95\% confluence in supplemented hormonal epithelial medium (SHEM, Sigma-Aldrich, Co., St Louis, MO) comprising $10 \%$ fetal bovine serum (FBS, Sigma-Aldrich, Co.). The cell culture was contained in a $75 \mathrm{~cm}^{2}$ culture flask and the cells were cultured at $35^{\circ} \mathrm{C}$ in a $5 \% \mathrm{CO}_{2}$ environment. We used SV40-HCECs passages 14-39 for our assays. After achieving the desired confluence, the cells were detached by incubating with $0.025 \%$ trypsin $/ 0.01 \%$ EDTA (Cascade Biologics, Portland, OR) for $10 \mathrm{~min}$ at $37^{\circ} \mathrm{C}$ and $5 \% \mathrm{CO}^{2}$ and then resuspended in $10 \mathrm{ml}$ of SHEM. SV40-HCECs were plated on the substrate chips at a density of 90,000 cells $/ \mathrm{ml}$ in SHEM and incubated for $24 \mathrm{~h}$ at $37{ }^{\circ} \mathrm{C}$ and in a $5 \% \mathrm{CO}_{2}$ environment.

\section{Transmission and Scanning Electron Microscopy Studies}

SEM imaged both the morphology and CSK of SV40-HCECs. Cell extraction preliminary to SEM of the CSK was conducted via a modification of previously reported methodology (Schliwa and van Blerkom, 1981). In this study we used PHEM buffer as a base for the extraction solution of the cells. The composition of PHEM buffer is 60mM PIPES (SigmaAldrich, Co.), 25mM HEPES (Sigma-Aldrich, Co.), 10mM EGTA (Sigma-Aldrich, Co.) and $2 \mathrm{mMMgCl}_{2}$ (Sigma-Aldrich, Co.) at a $\mathrm{pH}$ of 6.9. We prepared an extraction solution consisting of $1 \%$ triton X-100 and 4\% PEG (MW 40,000, Serva, Heidelberg, NY) in PHEM buffer supplemented with $10 \mu \mathrm{g} / \mathrm{ml}$ taxol (Sigma-Aldrich, Co.) and $10 \mu \mathrm{M}$ phalloidin (Sigma-Aldrich, Co.). After incubation, SV40-HCECs were rinsed with PBS and then 
incubated with the extraction solution for $3 \mathrm{~min}$ at room temperature with gentle agitation. The extracted cells were rinsed in $0.1 \mathrm{M}$ cacodylate buffer (Tousimis Corp., Rockville, MD) for $3 \times 10 \mathrm{~min}$ and fixed in $4 \%$ glutaraldehyde (Tousimis Corp.) in $0.1 \mathrm{M}$ sodium cacodylate buffer for $2.5 \mathrm{~h}$ and then rinsed again in cacodylate buffer. Next, the cells were post-fixed in $1 \%$ osmium tetroxide (Tousimis Corp.) in $0.1 \mathrm{M}$ sodium cacodylate for an hour. Post-

fixation was followed by dehydration through a graded series of ethanol concentrations (70, $80,90,99,100 \mathrm{vol} \%$ ) for at total of $15 \mathrm{~min}$ and a final dehydration step in hexamethyldisilazane (Sigma-Aldrich, Co.) for $5 \mathrm{~min}$. The samples were then air-dried, coated with $2 \mathrm{~nm}$ of platinum via ion beam sputtering (VCR IUS model 200) and imaged with a LEO 1530 SEM operating at an accelerating voltage of 1-5 kV.

TEM was used to image the CSK at the basal surface of SV40-HCECs cultured on the substrates with uniform groove ridge patterns. In this case, cells were prepared as described above. The samples were dehydrated through a series of ethanol (70-90 vol\%) and infiltrated with a 7:3 mixture of LR gold resin (London Resin Co. Ltd., Berkshire, UK) to ethanol for $3 \mathrm{~h}$ at $-27^{\circ} \mathrm{C}$. Then the cells were infiltrated further in pure resin for another $3 \mathrm{~h}$ and then embedded, at $-27^{\circ} \mathrm{C}$ and in UV light, using inverted gel capsules. The embedded cells were then separated from the substrates and the resulting resin blocks were cut into 70 $\mathrm{nm}$ thick sections (Reichert-Jung Ultracut- E, Leica, Deerfield, IL) and observed by TEM (JEOL 100CX, Peabody, MA). The location of grooves and ridges in the electron micrographs was determined by the thickness of the resin layer. Groove locations were marked by a darker coloring of the resin while ridge locations were lighter.

TEM was used to investigate the localization of immunogold-labeled $-\beta_{1}$ integrins at the undersurface of the extracted and nonextracted cells. In nonextracted cells, colloidal gold immunolabeling of $-\beta_{1}$ integrins was performed after incubation by first rinsing cells with PBS and then incubating cells with anti-human $-\beta_{1}$ integrin antibodies (CD29, Biosource International, Camarillo, CA) in the supplemented PHEM buffer at a dilution of 1:15 for half an hour. The cells were then rinsed in the supplemented PHEM buffer and then fixed in 4\% glutaraledhyde in PBS for 10 min. Next the cells were rinsed with PBS and blocked with $1 \%$ bovine serum albumin (BSA, Sigma-Aldrich, Co.) in a buffer containing $20 \mathrm{mM}$ Tris$\mathrm{HCl}$ (Fisher Scientific, Fair Lawn, NJ) at pH 8.0, 0.5 MNaCl (Sigma-Aldrich, Co.) and $0.05 \%$ Tween 20 (Fisher Scientific). The cells were incubated with the secondary antibody (donkey anti-mouse IgG conjugated to $18 \mathrm{~nm}$ gold particles, Jackson Immuno Research Laboratories) at $4{ }^{\circ} \mathrm{C}$ for $12 \mathrm{~h}$. The secondary antibody was applied at an approximate concentration of $8 \times 10^{11}$ particles per milliliter in a $20 \mathrm{mM}$ Tris- $\mathrm{HCl}$ solution at $\mathrm{pH} 8.0$ supplemented with $0.5 \mathrm{M} \mathrm{NaCl}, 0.05 \%$ Tween 20 and $0.1 \%$ BSA. Next, the cells were rinsed repeatedly with a buffer containing $20 \mathrm{mM}$ Tris- $\mathrm{HCl}$ at $\mathrm{pH} 8.0$ supplemented with $0.5 \mathrm{M}$ $\mathrm{NaCl}, 0.05 \%$ Tween 20 and $0.1 \mathrm{BSA}$. The final rinse step was followed by a fixation step in $4 \%$ glutraldehyde in $0.1 \mathrm{M}$ sodium cacodylate buffer for $5 \mathrm{~min}$, a post-fixation step in $1 \%$ osmium tetroxide in $0.1 \mathrm{M}$ sodium cacodylate buffer for $1 \mathrm{~h}$, dehydration in a graded ethanol series (70-90 vol\%), infiltration with resin and the embedding of the cells. Subsequent to embedding, the capsules were separated from the substrates and the resulting resin blocks were cut into $70 \mathrm{~nm}$ thick sections (Reichert-Jung Ultracut- E, Leica) and observed by TEM (JEOL 100CX). Colloidal gold immunolabeling of $-\beta_{1}$ integrins in extracted cells followed a similar procedure as that for nonextracted cells but was performed after cell extraction.

To control for nonspecific binding of imunoglobulins, labeling with the gold conjugated, donkey anti-mouse $\operatorname{IgG}$ was carried out with the substitution of PBS for the primary mouse anti-integrin antibody. No labeling was observed thus indicating that the donkey immunoglobulins did not exhibit significant nonspecific labeling. To be certain there was no nonspecific binding unique to mouse IgG, a fluoresceinated control mouse IgG (SigmaAldrich, Co.) was used in place of the primary antibody in the absence of blocking agents 
and second antibody. No fluorescence labeling was seen. Although the primary monoclonal antibody to the integrin is a mouse $\mathrm{IgG} 1$ we feel the $\mathrm{IgG} 2$ control antibody is sufficiently similar to rule out nonspecific mouse IgG binding as a concern.

\section{Results}

Silicon substrates fabricated by X-ray lithography had uniform groove and ridge features with nano- to microscale dimensions. The smallest patterns had a periodicity or pitch of 400 $\mathrm{nm}$ and pattern integrity and uniformity was achieved. Each feature size was in a $2 \times 2 \mathrm{~mm}^{2}$ area and a summary of the dimensions of the feature sizes in the substrates used in this study are shown in Table I. The groove to ridge ratio was approximately 1:1. The substrates used in this study are physically and chemically similar to substrates used in a previous study (Karuri et al. 2004) and therefore, we can relate the electron microscopy results obtained in this study to the morphology and adhesion results obtained in the previous studies.

SEM studies revealed that topography had an influence on both the cell shape and CSK organization in SV40-HCECs. A morphological response to the groove and ridge patterns was evident in cells adherent on nano- and microscale groove and ridge patterns (Fig. 1A(i) and (ii)). Cells on the patterns exhibited contact guidance; that is, they aligned and elongated in the direction of the anisotropic features. In contrast, cells cultured on the planar surface, represented by Figure 1A(iii), did not show this anisotropic morphological response. Scanning electron micrographs of the cell periphery yielded details on the organization of the CSK close to the underlying topography and demonstrated that the organization of the CSK in SV40-HCECs depended on topography. Cells on the 400 and 4,000 nm pitch (Fig. $1 \mathrm{~A}(\mathrm{iv})$ and (v)) had CSK elements that were oriented in the direction of the patterns. Cells on the planar surface exhibited a meshwork of CSK fibers crossing each other at the cell periphery (Fig. 1A(vi)).

Compared with cells on the nanoscale features, cells on the microscale grooves had larger and more distinct bundles of CSK elements. Scanning electron micrographs showed that on both the nano- and microscale patterns, the CSK bundles were formed preferentially on top of the ridges and fibers spanned the grooves (Fig. 1B(i) and (ii)). Cross-sectional micrographs of extracted cells demonstrated that cell-substrate contact was restricted to the tops of the ridges and that the width of the CSK fibers spanning the nanoscale grooves was smaller than that of fibers spanning the microscale grooves.

We obtained top down micrographs of the cells on the different feature sizes and measured the width of the CSK bundles spanning the grooves. Figure 2 represents a quantitative analysis of the width of the spanning fibers in cells cultured on the $400 \mathrm{~nm}$ and on the 1,200 and 4,000 $\mathrm{nm}$ pitch patterns and shows that the smaller the grooves the smaller the spanning CSK fibers. The groove to ridge ratio was similar across the different feature sizes and hence the spreading area was comparable on the different sized grooves. While both nanoand microscale grooved substrates induce the same extent of contact guidance in the overlying cells, the organization of the CSK in SV40-HCECs appears to be influenced by feature size.

Transmission electron studies of the basal surface of SV40-HCECs confirmed the SEM observations that showed that topography had an influence on the organization of the CSK. The basal CSK of SV40-HCECs cultured on the nano- and microscale grooves showed alignment with the direction of the patterns, but cells on the microscale features showed a more distinct organization of the CSK bundles (Fig. 3A and B). The position of the grooves and ridges near the basal surface was discernible from the thickness of resin and from the observation of serial sections near this surface. Transmission electron micrographs also 
supported the SEM finding that bundles formed preferentially on top of the ridges and fibers spanned the grooves. The smaller fibers having diameters of $10 \mathrm{~nm}$ or less primarily aligned with the features and comprised the bundles. The larger filaments having diameters $20 \mathrm{~nm}$ or more did not exhibit this same alignment. TEM studies on the CSK of cells cultured on the planar surface displayed a meshwork structure of filaments and bundles of filaments, similar to that shown in Figure 1(iv) (data not shown). Oblique sections of cells revealed that deeper in the cell bundles of CSK elements were aligned with the underlying patterns (Fig. 3C). This alignment shows some connectivity between the basal CSK and the inner CSK elements.

Immunogold labeling revealed that $-\beta_{1}$ integrins colocalized with microfilaments (MFs) at the basal surface of the SV40-HCECs and were concentrated at poles of spindle-shaped cells that exhibited contact guidance. We observed some association of $-\beta_{1}$ integrins with MFs although labeling was not extensive (Fig. 4A). To account for adhesion receptors that may have been removed or denatured during the extraction process, we performed immunogold labeling in nonextracted cells. In nonextracted, SV40-HCECs we observed a high concentration of $-\beta_{1}$ integrins on the cell membrane at the tips of elongated cells (Fig. 4B). Negative control experiments indicated that both primary and secondary antibody staining was specific (data not shown).

\section{Discussion}

Research into the effect of topography on cell behavior has demonstrated a morphological cellular response to nano- and microscale grooved substrates (Clark et al. 1990; Karuri et al. 2004; Teixeira et al. 2003). In a previous study, we reported that aligned and elongated HCECs on nanoscale groove and ridge patterns had more stable cell-substrate interactions than aligned and elongated cells on microscale groove and ridge patterns. Therefore, there may be decoupling of the morphological and adhesive response to the underlying substrate topography. In this study we establish that topography impacts SV40-HCEC spreading; the organization of the CSK elements and the distribution of $-\beta_{1}$ integrins. Nano- and microscale groove and ridge patterns cause the CSK of overlying cells to align with the patterns. However, there were distinct differences between CSK organization over nanoscale as opposed to the microscale grooves.

The periphery of aligned and elongated cells on the nano- and microscale features showed bundles of CSK elements located on top of ridges. The architecture of the CSK of aligned and elongated SV40-HCECs on microscale feature sizes contained larger and more distinct CSK bundles than in cells adherent to nanoscale features. Fibers spanned the grooves and interfaced with the bundles located preferentially on top of the ridges. The width of the fibers spanning the grooves was proportional to the width of the grooves. Fluorescent microscopy studies have shown that MFs and microtubules in cells cultured on microscale grooves align with the direction of the pattern (Oakley et al. 1997; Wojciak-Stothard et al. 1995). In this study we extend these findings to biological length scale or nanoscale patterns. We also demonstrate that organization of CSK elements is dependent on feature size.

While our study did not isolate how individual CSK components were influenced by topography, a qualitative analysis of TEM images of the basal CSK indicated that MFs tend to bundle on top of the ridges and align with the patterns. While cells cultured on the planar control surface also exhibited bundling of the basal CSK, the organization of the CSK did not exhibit the anisotropic organization observed in cells on the patterned features. Furthermore, cross-section studies of the CSK reinforced past studies that have demonstrated that cell-substrate interactions on similar features are limited to the top of the ridges (Karuri et al. 2004; Teixeira et al. 2003). Therefore, the physical features on the 
substrate may introduce adhesive and nonadhesive areas that influence the orientation and organization of CSK elements.

The extracellular matrix interfaces between the cell and its substrate, harbors ligands for cell-substrate adhesion and its organization is dependent on the CSK (Geiger et al. 2001). Through cell-extracellular matrix interactions such as spreading, cells stretch the extracellular matrix, expose cryptic matrix assembly sites and facilitate incorporation of new extracellular components into the developing structure. Changes in cell spreading and subsequent changes in CSK organization may translate into changes in the extracellular matrix in terms of amount of adhesive ligands present for adhesion. One possible outcome of these events is that the strength cell-substrate adhesion varies with topographical length scale as previously reported (Karuri et al. 2004).

Differences in the distribution of the areas available for cell spreading may also influence the adhesive response of SV40-HCECs. Adhesion receptors, $-\beta_{1}$ integrins, co-localized with the CSK elements at the basal surface of cells. This association is indicative of a connection between the CSK and cell-substrate adhesion. $-\beta_{1}$ integrins were concentrated in the poles of spindle-shaped aligned and elongated cells that displayed contact guidance indicating that the tips of the aligned and elongated cells may be areas of stronger cell-substrate attachments. In a previous study, we demonstrated that exposure of adherent SV40-HCECs to fluid shear in the direction of the underlying patterns led to a reduction in the percentage of aligned and elongated cells (Karuri et al. 2004). A difference in the local concentration of adhesion receptors within a cell may result in localized failure of cell-substrate linkages when a detachment force is applied.

This study presents evidence of a response to substrate topography at the level of cellular ultrastructure. Studies were conducted in serum-free media, which ensures that the contribution of growth factors and adhesive components in the serum such as fibronectin would be isolated. Future studies on the same lines as those presented in this report and incorporating other cues from the cell's natural environment would contribute significantly to the understanding of the interplay between chemical and physical factors regulating cellsubstrate adhesion.

\section{References}

Abrams GA, Schaus SS, Goodman SL, Nealey PF, Murphy CJ. Nanoscale topography of the corneal epithelial basement membrane and Descemet's membrane of the human. Cornea 2000;19:57-64. [PubMed: 10632010]

Abrams, GA.; Teixeira, AI.; Nealey, PF.; Murphy, CJ. Effects of substratum topography on cell behavior. In: Dillow, AK.; Lowman, AM., editors. Biomimetic Materials and Design. Marcel Dekker; New York: 2002. p. 91-137.

Chen CS, Mrksich M, Huang S, Whitesides GM, Ingber DE. Geometric control of cell life and death. Science 1997;276:1425-1428. [PubMed: 9162012]

Chou L, Firth JD, Uitto VJ, Brunette DM. Substratum surface topography alters cell shape and regulates fibronectin mRNA level, mRNA stability, secretion and assembly in human fibroblasts. J Cell Sci 1995;108(Pt 4):1563-1573. [PubMed: 7615675]

Clark P, Connolly P, Curtis AS, Dow JA, Wilkinson CD. Topographical control of cell behaviour: II. Multiple grooved substrata. Development 1990;108:635-644. [PubMed: 2387239]

Danen EH, Sonneveld P, Brakebusch C, Fassler R, Sonnenberg A. The fibronectin-binding integrins alpha5beta1 and alphavbeta3 differentially modulate RhoA-GTP loading, organization of cell matrix adhesions, and fibronectin fibrillogenesis. J Cell Biol 2002;159:1071-1086. [PubMed: 12486108]

Geiger B, Bershadsky A, Pankov R, Yamada KM. Transmembrane crosstalk between the extracellular matrix-cytoskeleton crosstalk. Nat Rev Mol Cell Biol 2001;2:793-805. [PubMed: 11715046] 
Hieda Y, Nakanishi Y. Epithelial morphogenesis in mouse embryonic submandibular gland: its relationships to the tissue organization of epithelium and mesenchyme. Dev Growth Differ 1997;39:1-8. [PubMed: 9079029]

Huang M, Spitz MR, Gu J, Lee JJ, Lin J, et al. Cyclin D1 gene polymorphism as a risk factor for oral premalignant lesions. Carcinogenesis 2006;27:2034-2037. [PubMed: 16638786]

Karuri NW, Liliensiek S, Teixeira AI, Abrams G, Campbell S, et al. Biological length scale topography enhances cell-substratum adhesion of human corneal epithelial cells. J Cell Sci 2004;117:3153-3164. [PubMed: 15226393]

Leivo I, Wartiovaara J. Basement membrane matrices in mouse embryogenesis, teratocarcinoma differentiation and in neuromuscular maturation. Int J Dev Biol 1989;33:81-89. [PubMed: 2485704]

Linsenmayer TF, Fitch JM, Gordon MK, Cai CX, Igoe F, et al. Development and roles of collagenous matrices in the embryonic avian cornea. Prog Retin Eye Res 1998;17:231-265. [PubMed: 9695794]

Oakley C, Brunette DM. The sequence of alignment of microtubules, focal contacts and actin filaments in fibroblasts spreading on smooth and grooved titanium substrata. J Cell Sci 1993;106(Pt 1):343-354. [PubMed: 8270636]

Oakley C, Jaeger NA, Brunette DM. Sensitivity of fibroblasts and their cytoskeletons to substratum topographies: topographic guidance and topographic compensation by micromachined grooves of different dimensions. Exp Cell Res 1997;234:413-424. [PubMed: 9260912]

Pankov R, Cukierman E, Clark K, Matsumoto K, Hahn C, et al. Specific beta1 integrin site selectively regulates Akt/protein kinase B signaling via local activation of protein phosphatase $2 \mathrm{~A}$. J Biol Chem 2003;278:18671-18681. [PubMed: 12637511]

Pankov R, Cukierman E, Katz BZ, Matsumoto K, Lin DC, et al. Integrin dynamics and matrix assembly: tensin-dependent translocation of alpha(5)beta(1) integrins promotes early fibronectin fibrillogenesis. J Cell Biol 2000;148:1075-1090. [PubMed: 10704455]

Schliwa M, van Blerkom J. Structural interaction of cytoskeletal components. J Cell Biol 1981;90:222-235. [PubMed: 7019221]

Teixeira AI, Abrams GA, Bertics PJ, Murphy CJ, Nealey PF. Epithelial contact guidance on welldefined micro- and nanostructured substrates. J Cell Sci 2003;116:1881-1892. [PubMed: 12692189]

Wojciak-Stothard B, Curtis AS, Monaghan W, McGrath M, Sommer I, et al. Role of the cytoskeleton in the reaction of fibroblasts to multiple grooved substrata. Cell Motil Cytoskeleton 1995;31:147158. [PubMed: 7553908]

Yamada S, Wirtz D, Coulombe PA. The mechanical properties of simple epithelial keratins 8 and 18 : discriminating between interfacial and bulk elasticities. J Struct Biol 2003;143:45-55. [PubMed: 12892725] 


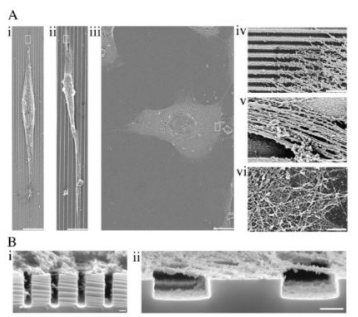

Fig 1.

Scanning electron micrographs reveal that topography influences morphology and cytoskeleton organization in SV40-HCECs. (A) Micrographs of adherent cells on the 400 $\mathrm{nm}$ pitch (i), $4 \mu \mathrm{m}$ pitch (ii) and planar surface (iii). High-magnification SEM micrographs of the outlined areas in (A) are represented by (iv) $400 \mathrm{~nm}$ pitch, (v) $4 \mu \mathrm{m}$ pitch and (vi) planar surface. Scale bar in (i), (ii) and (iii) $=10 \mu \mathrm{m}$. Scale bar in (iv), (v) and (vi) is $1 \mu \mathrm{m}$. (B) Scanning electron micrographs of cross-sections of the cytoskeleton of cells on the patterned substrates. (i) $400 \mathrm{~nm}$ pitch and (ii) $4 \mu \mathrm{m}$ pitch. Scale bar in (i) and (ii) is $100 \mathrm{~nm}$ and $1 \mu \mathrm{m}$, respectively. 


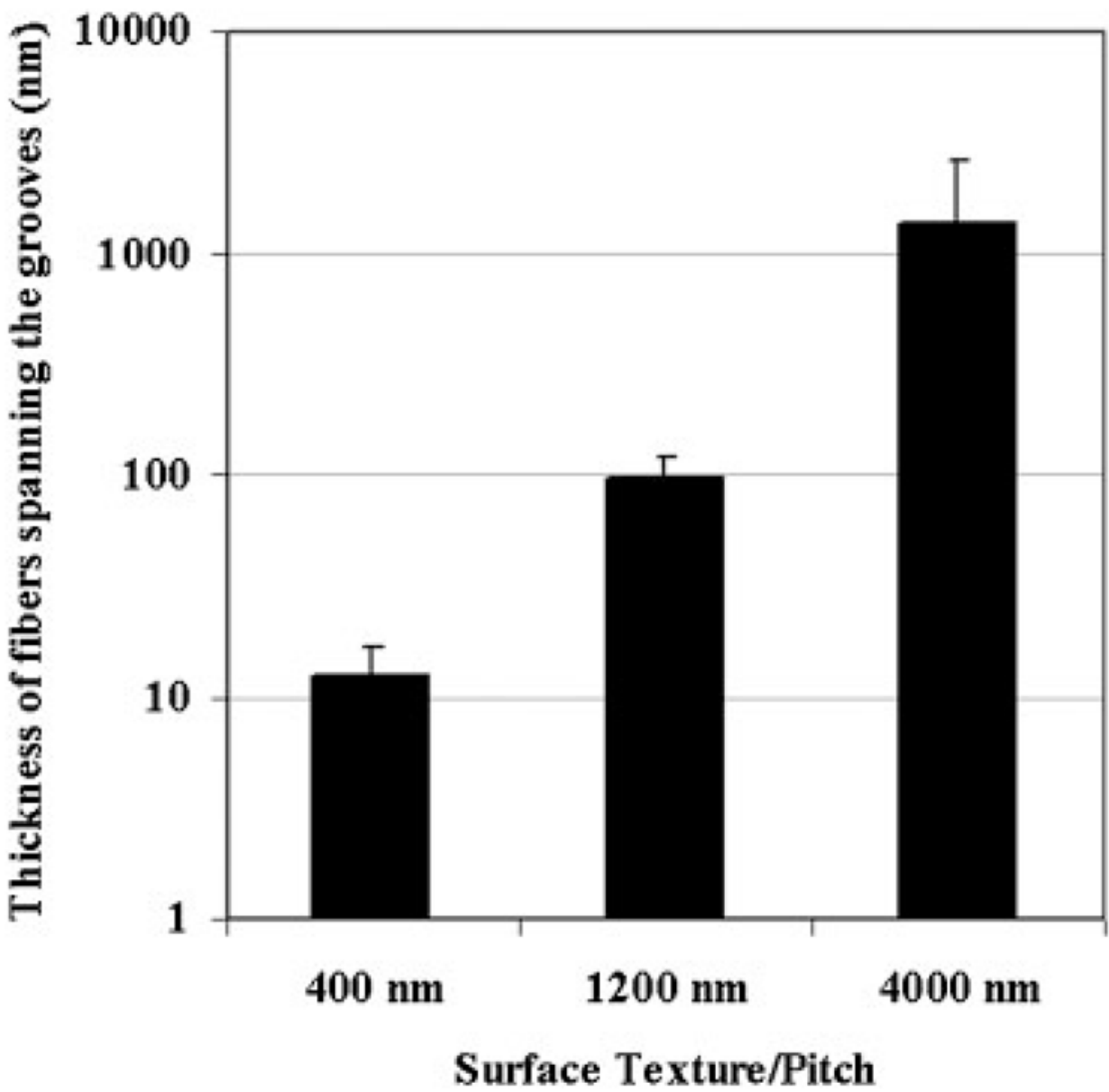

Fig 2.

Thickness of the fibers spanning the grooves depends on the groove size. The size of the spanning fibers was obtained from top down SEM measurements. SEM, scanning electron microscopy. 

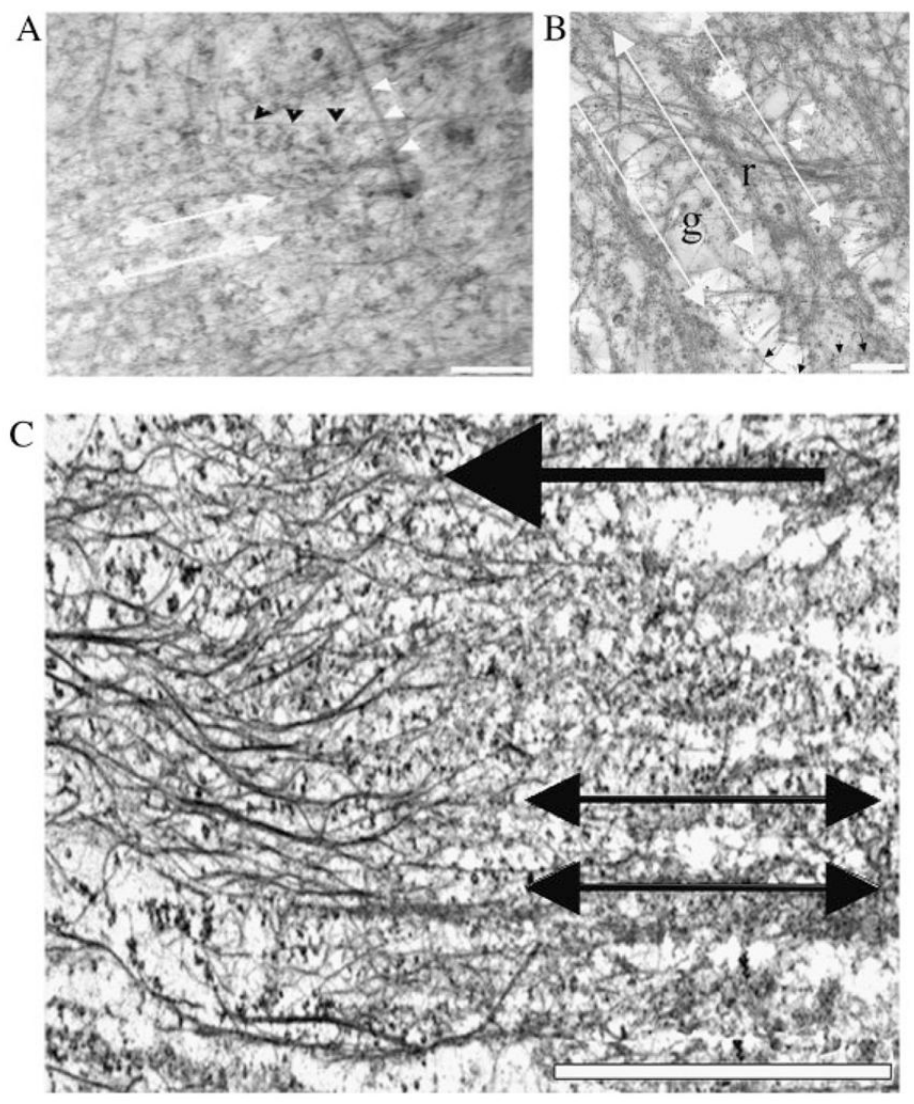

Fig 3.

Transmission electron micrographs show that topography influences the organization of the basal cytoskeleton as well as cytoskeleton deeper in the cell. Sections of the basal surface of an SV40-transformed HCEC cultured on the $400 \mathrm{~nm}$ pitch (A) and on the 1,200 nm pitch (B). An oblique section of a cell on the $1,200 \mathrm{~nm}$ pitch is shown in (C). The double-headed white arrows in (A), (B) and (C) show the direction of the patterns. The short white arrows and the short black arrows in (A) and (B) indicate the position of microtubules and intermediate filaments, respectively. The letters "g" and " $r$ " in B mark the groove and ridge locations, respectively. The red arrow points to the direction of increasing depth from the basal surface; that is, the cytoskeletal elements on the left side of the image are further away from the basal surface of the cell than those on the right side (tilt angle is approximately $2^{\circ}$ ). Scale bar in (A) and (B) is $500 \mathrm{~nm}$. Scale bar in (C) is $5 \mu \mathrm{m}$. HCEC, human corneal epithelial cell. 


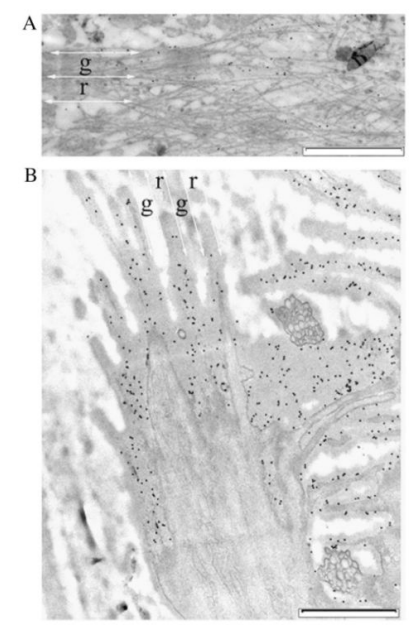

Fig 4.

$-\beta_{1}$ integrins at the basal surface of the cell are associated with the cytoskeleton and have a higher density at the apex of aligned and elongated cells. (A) The cytoskeleton of SV40HCEC on $400 \mathrm{~nm}$ pitch is associated with $-\beta_{1}$ integrins. (B) Micrograph showing distribution of $-\beta_{1}$ integrins on the undersurface of a nonextracted SV40-HCEC on the 400 $\mathrm{nm}$ pitch. Scale bar in (A) and (B) is $1 \mu \mathrm{m}$. 


\section{Table I}

Average surface dimensions of the groove and ridge structures

\begin{tabular}{lcc}
\hline Surface & Ridge size $(\mathbf{n m})$ & Groove size $(\mathbf{n m})$ \\
\hline $400 \mathrm{~nm}$ pitch & $222 \pm 21$ & $176 \pm 19$ \\
$800 \mathrm{~nm}$ pitch & $453 \pm 18$ & $344 \pm 18$ \\
$1,200 \mathrm{~nm}$ pitch & $680 \pm 52$ & $511 \pm 54$ \\
$1,600 \mathrm{~nm}$ pitch & $932 \pm 31$ & $664 \pm 29$ \\
$2,000 \mathrm{~nm}$ pitch & $1,133 \pm 26$ & $863 \pm 36$ \\
$4,000 \mathrm{~nm}$ pitch & $2,150 \pm 57$ & $1,835 \pm 51$ \\
\hline
\end{tabular}

The average depth was approximately $400 \pm 150 \mathrm{~nm}$; owing to diffusion limitations in the etching process, the large pitches had higher depth values. Mean \pm s.e.m. $n=5$. 